Research Article

\title{
Beam Scanning Properties of a Ferrite Loaded Microstrip Patch Antenna
}

\author{
Sheikh Sharif Iqbal Mitu and Farooq Sultan \\ Electrical Engineering Department, King Fahd University of Petroleum and Minerals, Dhahran 31261, Saudi Arabia \\ Correspondence should be addressed to Farooq Sultan; farooqsultan@kfupm.edu.sa
}

Received 25 May 2014; Revised 22 August 2014; Accepted 21 September 2014

Academic Editor: Diego Caratelli

Copyright (C) 2015 S. Sharif Iqbal Mitu and F. Sultan. This is an open access article distributed under the Creative Commons Attribution License, which permits unrestricted use, distribution, and reproduction in any medium, provided the original work is properly cited.

\begin{abstract}
Axially magnetized ferrite loaded microstrip patch antenna (MPA) with tunable beam scanning properties is presented. Ferrite cylinders are optimally positioned within the near field region of the patch to introduce $E_{y}$ phase tapers needed for beam scanning. The interaction between the radiated EM wave and the gyrotropic properties of ferrites is controlled by varying the magnetizing fields. A beam scan of $\pm 30^{\circ}$ is achieved for a DC biasing range of 0-0.19 T. Simulated antenna properties are verified using experimental results. Recent LTCC technology allows the biasing coils to be embedded within the ferrite material to considerably reduce the required external magnetizing field.
\end{abstract}

\section{Introduction}

In recent wireless sensor and communication systems, antennas with beam scanning capability are of great interest to achieve reconfigurable coverage $[1,2]$. Printed phased array antennas (PAA) are widely used in applications like target tracking and interference avoidance, where a costly and lossy network of phase shifters are needed to realize externally controllable directive beam scan [3]. The narrow half power beam width (HPBW) of a PAA can be a limiting factor for the beam scannable sector antennas used in RFID, GPS, and WLAN applications. Although microstrip patch antennas (MPA) with wider HPBW are more suited for the above mentioned applications, they lack the capability of beam scanning.

In the literature, beam steering of MPA is investigated by Thongsopa et al. [4], where beam steering of a dual feed patch antenna depends on the difference between the input feed frequencies. Ha and Jung [5] have presented a wearable patch antenna, where main beam can be switched between $0^{\circ}$ and $\pm 30^{\circ}$. Attia et al. in [6] have achieved beam steer by using a specially designed superstrate layer. Cao et al. in [7] have achieved a maximum beam steer of $48^{\circ}$ by introducing complementary split ring resonators (CSRR) in the ground plane of the MPA. Since the above techniques failed to scan the main beam in a continuous manner, a ferrite loaded microstrip patch antenna is proposed here with tunable beam scanning characteristics.

When magnetized, gyrotropic properties of microwave ferrites are expressed using tensor permeability [8]. In the literature, ferrite phase shifters have been widely used to produce the progressive phase shift required to control the beam steering characteristics of microstrip phased array antennas [9]. As far as the single MPAs are concerned, interaction of the RF signal with magnetized ferrite material has been used for antenna miniaturization [10-13], widening the impedance bandwidth of the antennas [14-16], and frequency tuning [17-19]. One of the first attempts to use magnetized ferrite for beam switching involved putting ferrite rods inside a horn antenna [20]; the design resulted in a change in the main beam direction by $\pm 22^{\circ}$. A number of antenna designs based on leaky wave cavities [21] and waveguide antennas [22] have been proposed where considerable beam scans have been achieved. The comprehensive literature search did not reveal any ferrite based beam scanning printed MPAs. A novel beam scanning technique for a single microstrip patch antenna (MPA) is presented here, where magnetized ferrite rods are optimally placed in the radiation region to control the $E_{y}$ phase distribution of the radiated field. Professional 


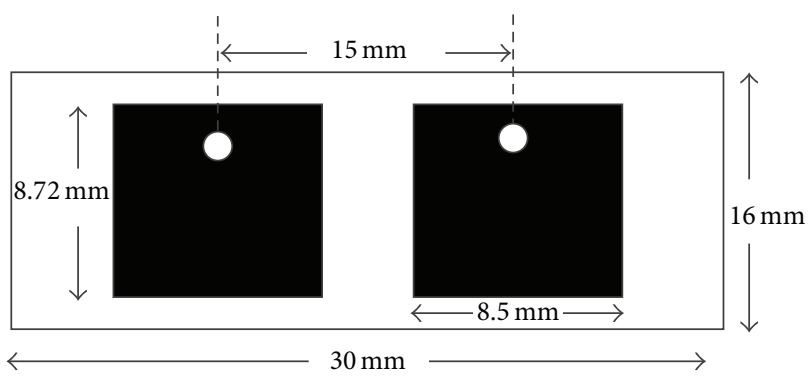

(a)

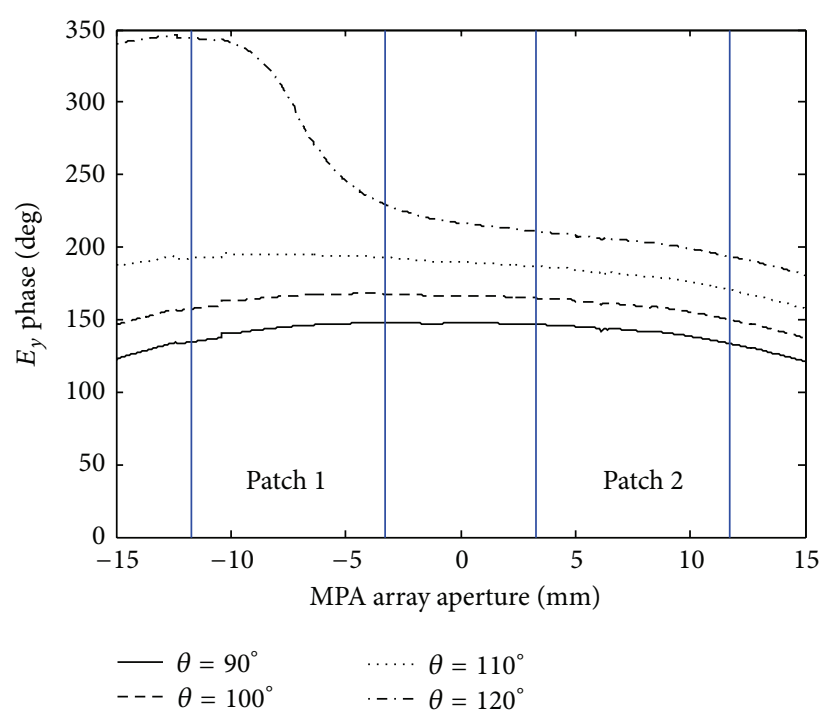

(b)

Figure 1: (a) Top view of a 2-patch $0.5 \lambda$ PAA operating at $10 \mathrm{Ghz}$, (b) $E$-field phase distribution in the radiated field region of the 2-element PAA for different directions of the main beam.

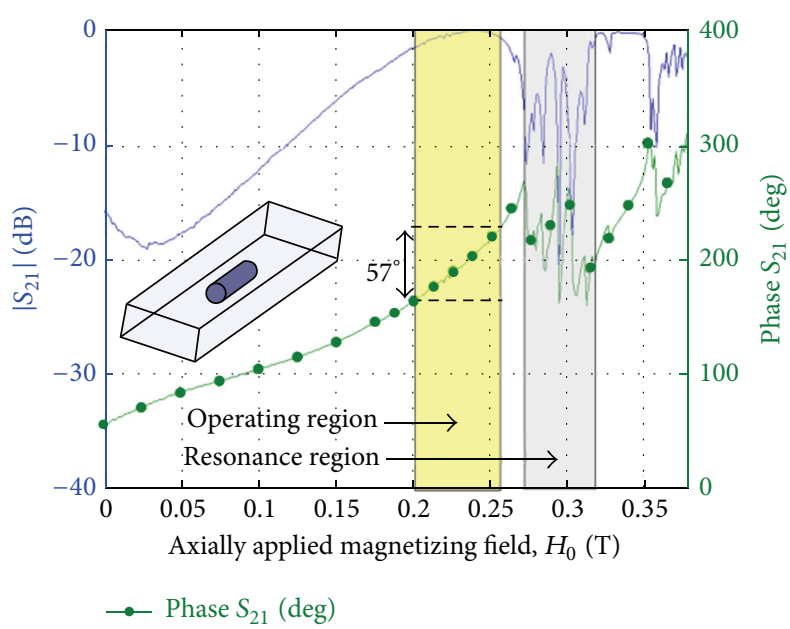

FIGURE 2: Magnitude and phase of the transmitted signal through a ferrite cylinder when excited by plane waves in a rectangular waveguide.

simulator (HFSS) is used to demonstrate the wide HPBW and externally tunable scanning capabilities of the designed antenna, ideal for sectorwise coverage of communication devices. The optimized antenna is fabricated and tested to verify the simulated reflection and radiation properties.

\section{Beam Scan Properties of Magnetized Ferrite Rods}

Beam scanning for linear PAA is achieved by introducing a progressive phase shift $(\beta)$ into the excitation signal of the individual elements [3]. This progressive phase shift results in a phase taper across the PAA aperture leading to a change in the direction of the main beam. For a two-element linear microstrip PAA with $d=0.5 \lambda$ operating at $10 \mathrm{GHz}$, the phase distributions of the radiated $E_{y}$-fields are shown in Figure 1.

Note that Figure 1(b) plots the $E_{y}$ phase distribution resulting from a change in the direction of the main beam $(\theta)$ for $\theta=90^{\circ}$ (broadside), $100^{\circ}, 110^{\circ}$ and $120^{\circ}$, realized by selecting progressive phase excitation with $\beta=0^{\circ}, 31.3^{\circ}, 611^{\circ}$ and $90^{\circ}$, respectively [3]. For a single MPA, $\beta$ does not apply; hence, to scan the main beam, the same amount of phase taper has to be produced in the radiated signal by some other mechanisms.

Magnetized ferrite is known to affect the magnitude and phase of the transmitting RF signal if properly aligned. To understand the phase control properties of ferrite, a Y220 ferrite cylinder with $r=6 \mathrm{~mm}, l_{f}=20 \mathrm{~mm}, \varepsilon_{r}=15.4$, $4 \pi M_{s}=1950$ Gauss, and $\Delta H=10$ Oe was placed in the path of propagating plane waves (inside a WR110 rectangular waveguide with a cutoff of $6.4 \mathrm{GHz}$ ) and the properties of the transmitted signal were observed. For an operating frequency of $10 \mathrm{GHz}$, the simulated (HFSS) magnitude and phase of the transmitted signal as a function of the biasing $\left(H_{0}\right)$ are plotted in Figure 2. Note that as the biasing $\left(H_{0}\right)$ starts to increase the magnitude of the transmitted signal starts to increase and becomes maximum at $0.245 \mathrm{~T}$. A low attenuation operating region (yellow shaded) has been indicated in Figure 2; changing the external magnetizing field $\left(\Delta H_{0}\right)$ by $0.048 \mathrm{~T}$ in this low-loss operating region results in a $57^{\circ}$ change in the insertion phase $(\Delta \phi)$ while maintaining maximum transmission throughout. This property of the ferrite to affect the phase of the passing RF signal can be used to create a phase taper similar to the one shown in Figure 1(b) that would cause beam scan. In the above resonance region, a phase 


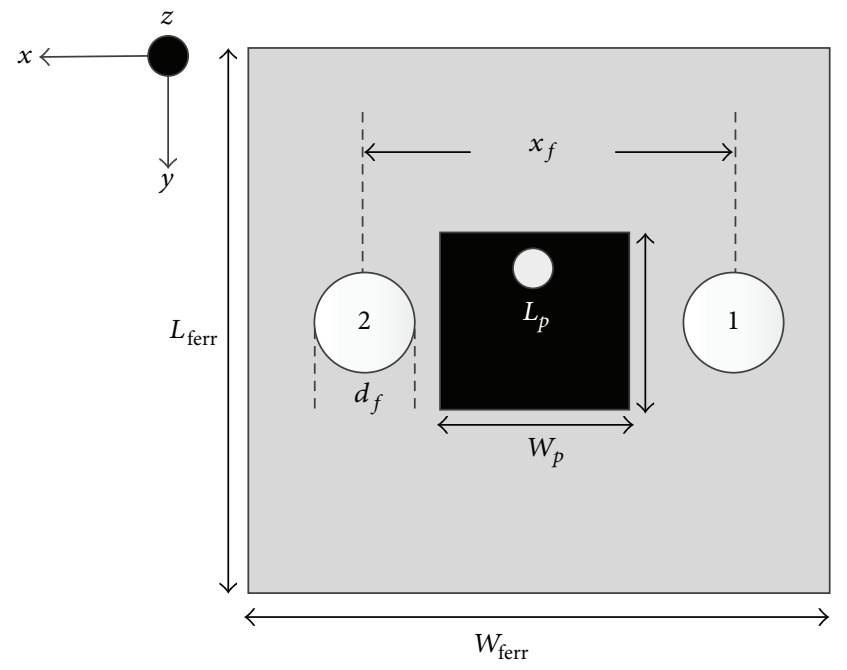

(a)

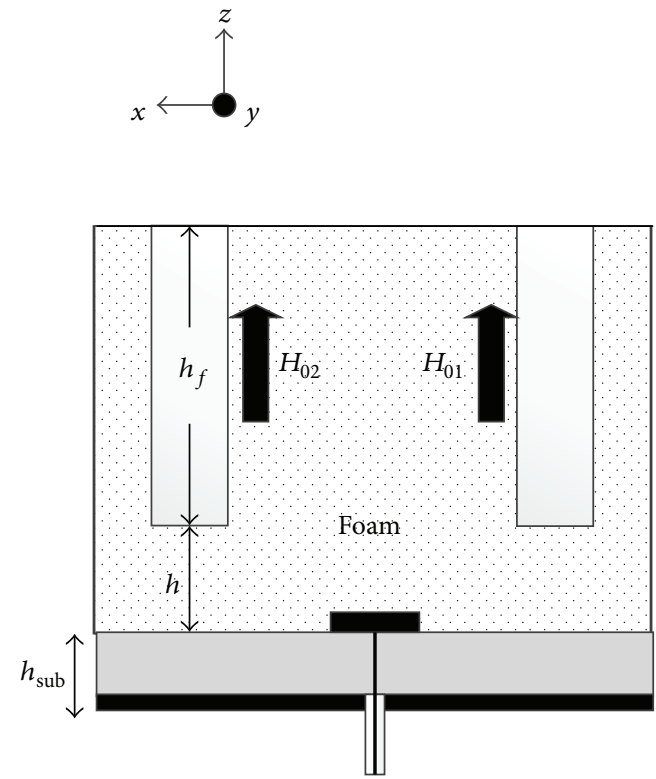

(b)

FIgURE 3: Magnetized ferrite loaded patch antenna: (a) top view and (b) side view.

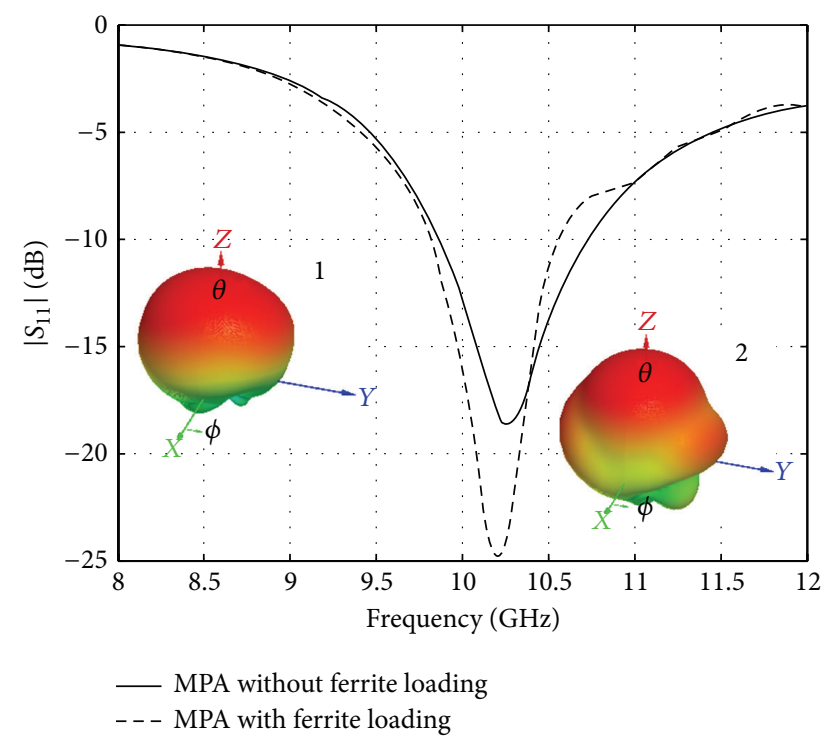

Figure 4: Simulated $S_{11}$ curves for the MPA with and without the ferrite superstrate.

change of nearly $100^{\circ}$ can be achieved by changing $H_{o}$ from $0.3147 \mathrm{~T}$ to $0.3513 \mathrm{~T}$.

\section{Design of Ferrite Loaded Patch Antenna}

The schematic diagram of the designed microstrip patch antenna (MPA) loaded with two separately magnetized ferrite rods is shown in Figure 3. In order to produce a beam scan in the azimuth plane, a phase taper in the $E$-field along the $x$ axis (Figure 3 ) has to be produced. It has been observed that

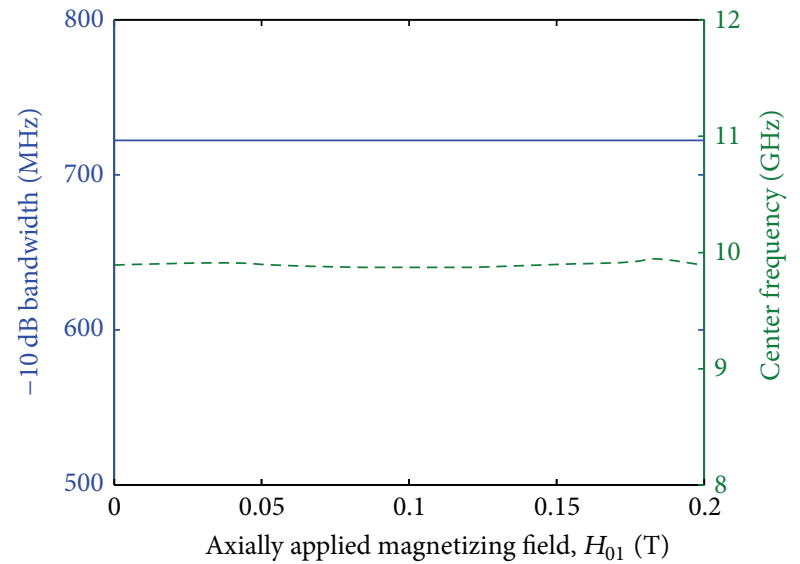

FIGURE 5: Simulated impedance bandwidth ( $-10 \mathrm{~dB}$ bandwidth) and center frequency for the ferrite loaded MPA for varying biasing $H_{01}$.

$E_{y}$ becomes the dominant component of the radiated field along the $x$-axis and the $x$ - and $y$-components of the $E$-field are very low in magnitude. Thus a phase taper in the $E_{y}$ is desired for a beam scan in the $E$-plane. Since a phase taper in the $x$-axis is needed, two ferrite rods have been placed along the $x$-axis on either side of the radiating patch. As already observed from Section 2, magnetized ferrite rod can change the phase of the transmitting signal; hence, biasing one of the ferrite rods at a time would decrease the phase of the signal passing through it resulting in a phase taper across the $x$-axis, leading to a beam scan.

3.1. Microstrip Patch Antenna Design. The MPA is designed on a Duroid substrate with $\varepsilon_{r}=2.2, t=1.6 \mathrm{~mm}$. Professional software (HFSS) is used to optimize the designed coaxially 


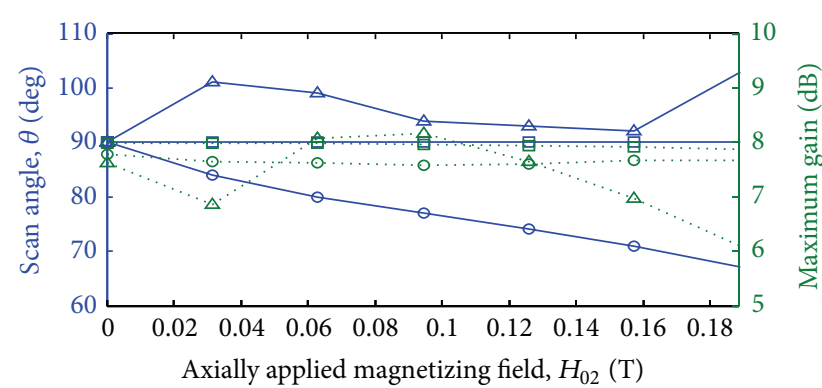

$$
\begin{aligned}
& \square d_{f}=3 \mathrm{~mm} \\
& \circ d_{f}=6 \mathrm{~mm} \\
& \Delta d_{f}=9 \mathrm{~mm}
\end{aligned}
$$

(a)

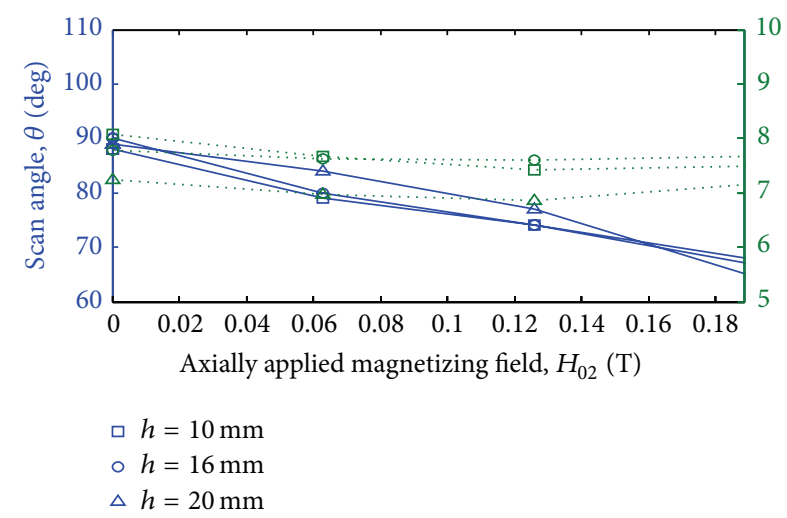

(c)

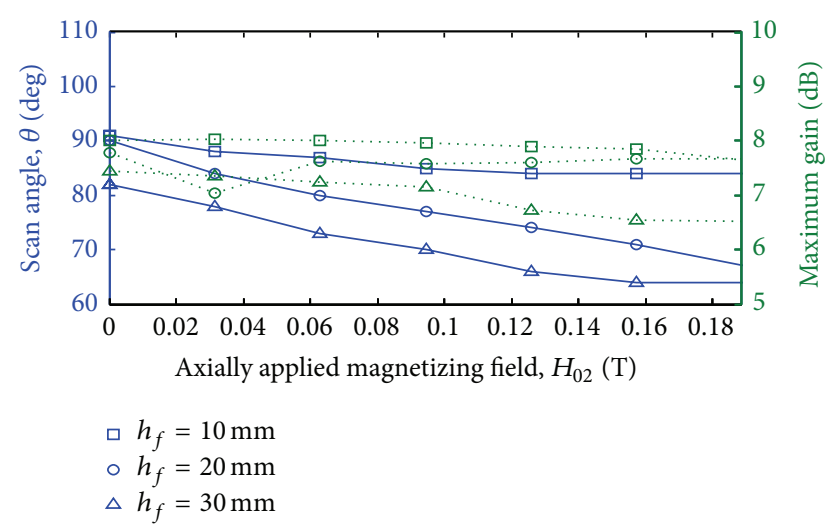

(b)

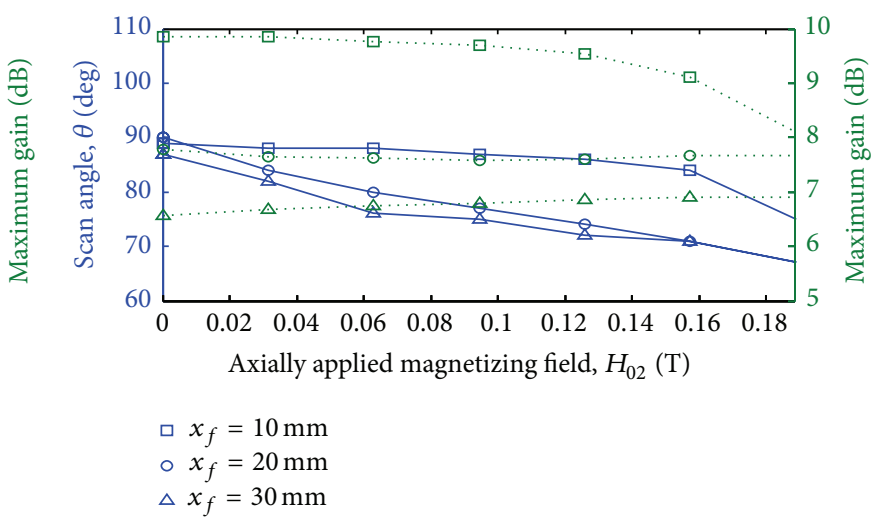

(d)

Figure 6: For $H_{01}=0$, the changes in beam scan angle (degrees) and the maximum gain are plotted for different values of (a) $d_{f}$, (b) $h_{f}$, (c) $h$, and (d) $x_{f}$. Dotted green lines represent the gain and the solid blue lines represent the scan angle.

fed patch to resonate at $10 \mathrm{GHz}$. The optimized dimensions of the MPA are $h_{\text {sub }}=1.6 \mathrm{~mm}, W_{\text {ferr }}=40 \mathrm{~mm}, L_{\text {ferr }}=40 \mathrm{~mm}$, $W_{p}=8.5 \mathrm{~mm}$, and $L_{p}=8.72 \mathrm{~mm}$. The ferrite rods are then loaded onto the MPA to introduce beam scanning. Figure 4 plots the simulated reflection response $\left(S_{11}\right)$ of the antenna, with and without ferrite loading. The 3D directivity patterns are also included; pattern 1 corresponds to the MPA without ferrite loading and pattern 2 corresponds to MPA with ferrite loading. Note that loading ferrite rods reduced the impedance bandwidth of the antenna by $1.2 \%$ (to $723.7 \mathrm{MHz}$ ). Moreover, changing the DC biasing fields $\left(H_{01}\right.$ or $\left.H_{02}\right)$, to realize beam scan, has no effect on the reflection characteristics as the impedance bandwidth and the center frequency remain unchanged. This is shown in Figure 5.

3.2. Placement and Dimension of the Ferrite Rods. The parameters related to the physical dimensions of the ferrite rods, shown in Figure 3, are discussed in this section. To facilitate beam steer in $\pm \theta^{\circ}$ angles from the broad side direction, the ferrite rods are separately biased using magnetizing fields, $H_{01}$ and $H_{02}$. With $H_{01}=0$ or unbiased, changing $H_{02}$ values steered the main beam of the MPA towards $-\theta$ angles $(\theta=$ $\left.80^{\circ}, 70^{\circ}, \ldots\right)$. Alternatively, by changing the magnetizing field $H_{01}$, the main beam is observed to steer towards $+\theta$ angles $\left(\theta=100^{\circ}, 110^{\circ}, \ldots\right)$. Selecting correct magnetizing field $\left(H_{0}\right)$, position $\left(x_{f}, h\right)$, and the dimensions $\left(d_{f}, h_{f}\right)$ of these ferrite rods is critical to achieve optimum gain and beam scanning properties. A professional simulator (HFSS) is used to carry out a comprehensive parametric analysis of the four variables, $x_{f}, h, d_{f}$, and $h_{f}$.

Setting $H_{01}=0$, the simulated parametric sweep with increasing values of $H_{02}$ is plotted in Figure 6. It is observed that beam scan depended on the difference between $H_{01}$ and $H_{02}$ and remains in the broadside direction with both ferrites unbiased $\left(H_{01}=H_{02}=0\right)$. Figure 6(a) plots the changing radiation parameters of the antenna with varying diameter of the ferrite rods $\left(d_{f}\right)$. For fixed values of the height $\left(h_{f}=20 \mathrm{~mm}\right)$ and separation $\left(x_{f}=20 \mathrm{~mm}\right)$, increasing the diameter to $6 \mathrm{~mm}$ changes the scan angle by $\Delta \theta=-26^{\circ}$ for a differential magnetizing field of $\Delta H_{02}=0.19 \mathrm{~T}$. Note that the gain of the antenna remains constant for the whole range of the biasing field. A further increase in $d_{f}$ reduced the antenna gain without improving the scan angle. Considering the parametric sweep for ferrite length $\left(h_{f}\right)$ in Figure 6(b), it is clear that a taller ferrite rod offers more beam scan compared to shorter ones. However, this also increases the antenna dimension in addition to reducing the antenna gain for a given value of $H_{02}$. Note that the optimum value, $h_{f}=$ $20 \mathrm{~mm}$, produces a maximum beam scan of $-26^{\circ}\left(\theta=64^{\circ}\right)$ 


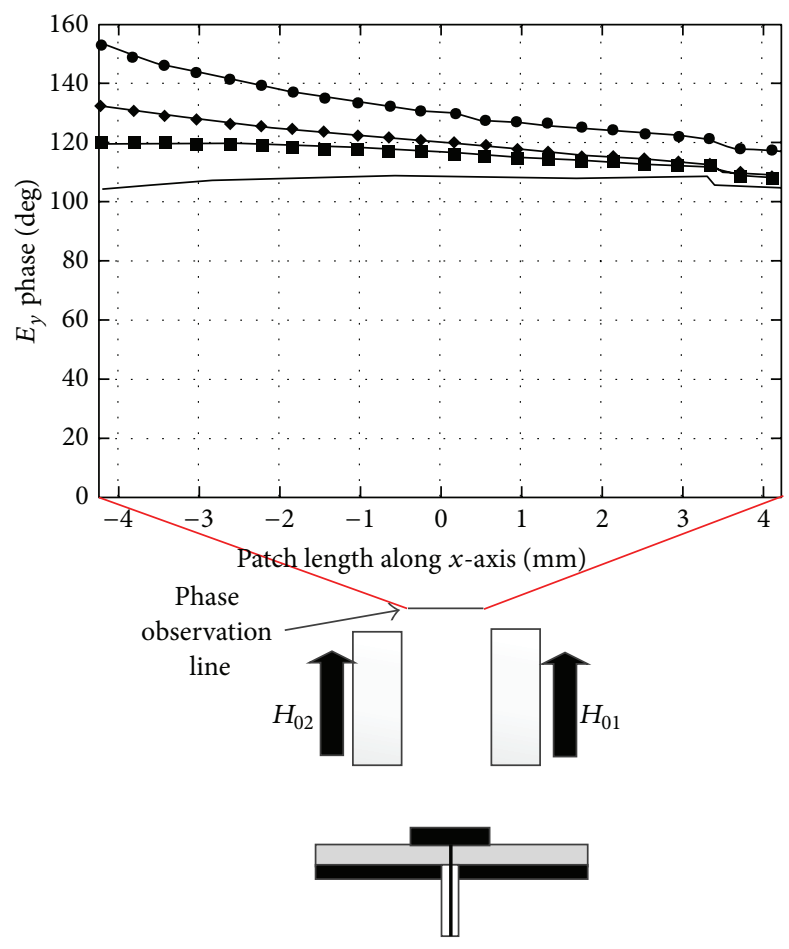

$$
\begin{aligned}
& \longrightarrow \theta=90^{\circ}, H_{01}=0 \mathrm{~T}, H_{02}=0 \mathrm{~T} \\
& \rightarrow \theta=80^{\circ}, H_{01}=0 \mathrm{~T}, H_{02}=0.109 \mathrm{~T} \\
& \multimap \theta=70^{\circ}, H_{01}=0 \mathrm{~T}, H_{02}=0.179 \mathrm{~T} \\
& \longrightarrow \theta=60^{\circ}, H_{01}=0 \mathrm{~T}, H_{02}=0.225 \mathrm{~T}
\end{aligned}
$$

FigURE 7: $E_{y}$ phase distribution in the NF region for different scan angles.

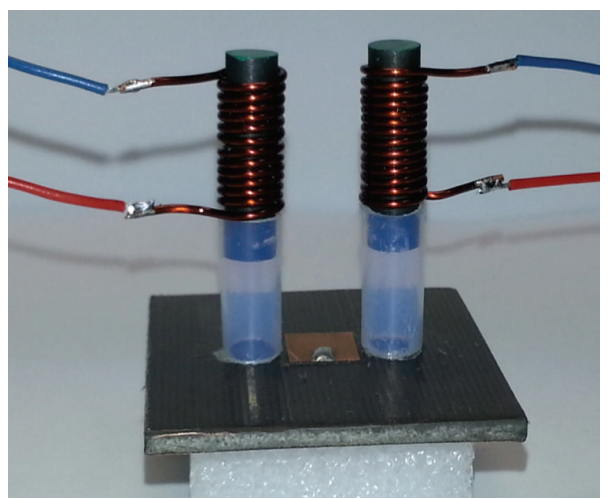

(a)

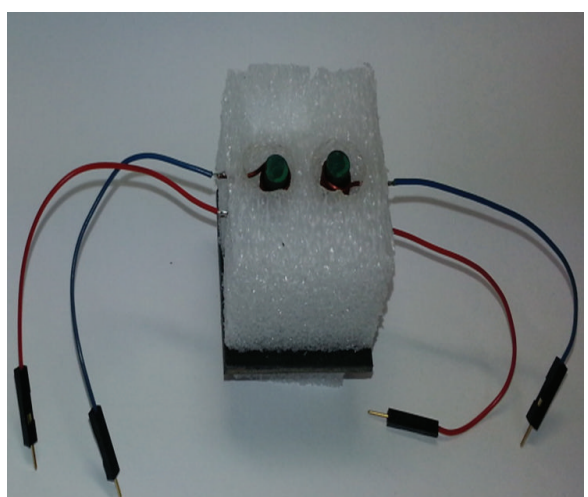

(b)

FIGURE 8: Fabricated $10 \mathrm{GHz}$ MPA loaded with ferrite rods, (a) ferrite rods with biasing coils and (b) with Styrofoam packing.

from broadside for $\Delta H_{02}=0.19 \mathrm{~T}$, where the antenna gain remains nearly constant at $7.8 \mathrm{~dB}$. Figure $6(\mathrm{c})$ clearly indicates that increasing the spacing " $h$ " between the patch surface and the ferrite rod tends to decrease the scan angle. This is due to the formation of the partially resonant cavity between the substrate and the ferrite rod, which supports additional modes. The parameter $h=16 \mathrm{~mm}$ is chosen, as it offers a good beam scan with best antenna gain throughout the sweep $\left(\Delta H_{02}\right)$. Optimum positioning of ferrite rods can facilitate more interaction between the ferrites and radiated EM waves to maximize the phase taper. In Figure 6(d), it is clear that placing the ferrite rods closer to the nonradiating edges of MPA provides higher beam scans at the cost of reduced antenna gain. Note that $x_{f}=20 \mathrm{~mm}$ provides the best 


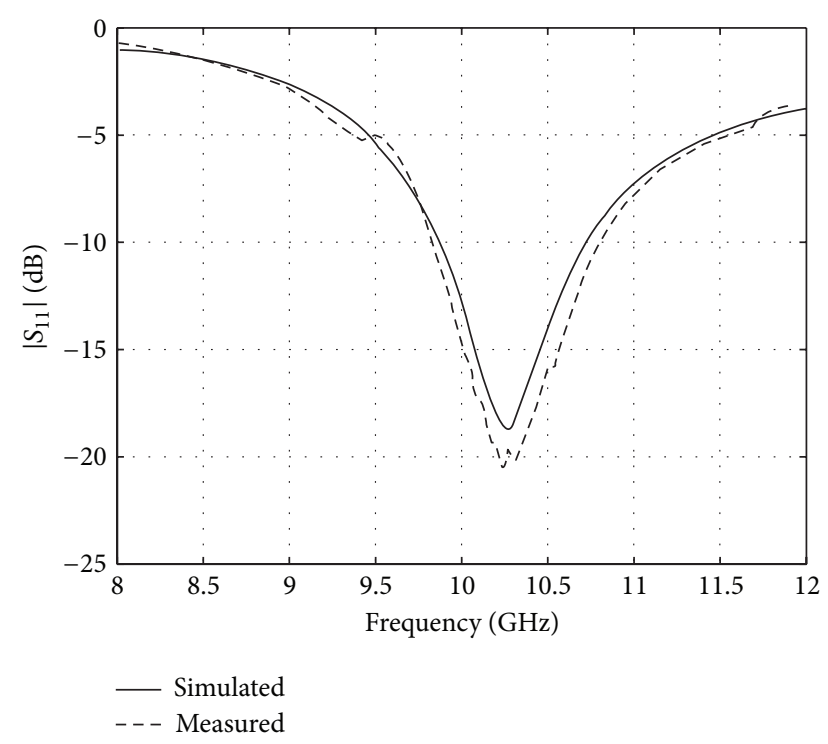

FIGURE 9: Simulated and experimental $S_{11}$ responses of the ferrite loaded MPA.

compromise between the scan angle and antenna gain for the whole range of $H_{02}$. Thus, the optimized antenna parameters that resulted in best combination of scan angle and antenna gain are $h_{f}=20 \mathrm{~mm}, x_{f}=20 \mathrm{~mm}, d_{f}=6 \mathrm{~mm}$, and $h=16 \mathrm{~mm}$. To achieve opposite directional beam scan, opposite biasing scheme is needed with unbiased $\mathrm{H}_{02}$ and increasing values of $H_{01}$ from 0 to 1 .

\section{Results}

The simulated $E_{y}$ phase distribution across the patch is plotted in Figure 7 for $H_{01}=0$ and four different values of $H_{02}$. Note that, for changing the biasing field $H_{02}$ by $\Delta H_{02}=$ $0.225 \mathrm{~T}$, the $E_{y}$ phase distribution changes by approximately $38^{\circ}$, which steers the main beam of the MPA by $\Delta \theta=$ $-25^{\circ}$ (or $\theta=65^{\circ}$ ). Similarly, to scan the main beam from broadside to $\theta=120^{\circ}$, the ferrite rods needed to be biased with $H_{02}=0$ and $\Delta H_{01}=0.225 \mathrm{~T}$. Figure 8 shows the prototype of the fabricated antenna. The coils that use variable DC current sources to axially magnetize the ferrite rods are also shown in the figure. Using Tesla meter, the ferrite filled coils are precalibrated to relate the coil currents with induced magnetizing fields. For packaging purposes, the positions of the ferrite rods are secured by a Styrofoam $\left(\varepsilon_{r} \approx 1\right)$ cube, as shown in Figure 8(b). This also allows the removal of the plastic pipes, used to position the ferrite rods in Figure 8(a).

A vector network analyzer is used to measure the reflection response $\left(S_{11}\right)$ of the ferrite loaded antenna. Figure 9 shows the simulated and experimental $S_{11}$ responses of the ferrite loaded microstrip patch antenna (MPA). It is observed that separately magnetizing ferrites have no effects on the impedance bandwidth and the resonance of the antenna. Using an antenna measurement setup, the beam
TABLE 1: Measured HPBW and directivity for designed antenna with $H_{01}=H_{02}=0$.

\begin{tabular}{lcc}
\hline & HPBW $(\mathrm{deg})$ & Directivity $(\mathrm{dB})$ \\
\hline $\begin{array}{l}\text { MPA without superstrate } \\
\begin{array}{l}\text { MPA with ferrite superstrate, } \\
\text { without biasing coils }\end{array}\end{array}$ & 81 & 6.687 \\
$\begin{array}{l}\text { MPA with ferrite superstrate, } \\
\text { with biasing coils }\end{array}$ & 83 & 7.139 \\
\hline
\end{tabular}

scanning properties of the designed antenna are experimentally observed.

A comparison of the measured and simulated radiation patterns of the antenna with $H_{01}=0$ and changing values of $H_{02}$ is plotted in Figure 10. Note that maximum simulated scan angles of $\pm 30^{\circ}$ are verified by the measured radiation patterns at $+28^{\circ}$ and $-26^{\circ}$, respectively. Higher back lobes and minor mismatch between simulated and experimental patterns are due to unwanted reflections normally eliminated by the anechoic chamber. It can be observed from Figure 10(a) that, for no beam scan case, the maximum measured directivity is $7.097 \mathrm{~dB}$ and the respective measured directivities at $64^{\circ}$ and $118^{\circ}$ are $5.255 \mathrm{~dB}$ and $6.069 \mathrm{~dB}$. It must be noted that inclusion of ferrite rods and the biasing coils has minimal effect on the radiation properties of the designed antenna and the measured HPBW and directivity values for the MPA without superstrate, with only ferrite, and with ferrite and biasing coils are provided in Table 1. When scanning the main beam to either of the maximum scan angles, $64^{\circ}$ (Figure $10(\mathrm{~b})$ ) and $118^{\circ}$ (Figure 10(c)), the peak directivity values measured at the respective directions of maximum are $6.69 \mathrm{~dB}$ and $6.53 \mathrm{~dB}$. Additionally the directivity measured in the original direction of maximum $\left(90^{\circ}\right)$ during beam scanning is $3.091 \mathrm{~dB}$ and $5.342 \mathrm{~dB}$ for $64^{\circ}$ and $118^{\circ}$, respectively. Although this antenna may seem bulky, using recent embedded windings technology available for LTCC ferrite devices can reduce the height and biasing requirement of the antenna by $95 \%$ [19].

\section{Conclusion}

Beam scanning characteristics of a ferrite loaded single MPA are presented. Using this novel method, ferrite rods are placed in the radiation region of the antenna to perturb the $E$ field phase distribution resulting in beam scan. Parametric analysis resulted in finding the optimum dimension, location, and biasing requirements of the ferrite rods. A simulated beam scan of $\Delta \theta= \pm 30^{\circ}$ is achieved for a changing biasing field of $\Delta H_{0}=0.214 \mathrm{~T}$. The designed antenna is fabricated to experimentally observe a beam scan of $+28^{\circ}$ and $-26^{\circ}$ for predicted changes of external magnetizing fields. Using recent LTCC ferrite techniques, the requirements of biasing field can be reduced by $95 \%$.

\section{Conflict of Interests}

The authors declare that there is no conflict of interests regarding the publication of this paper. 


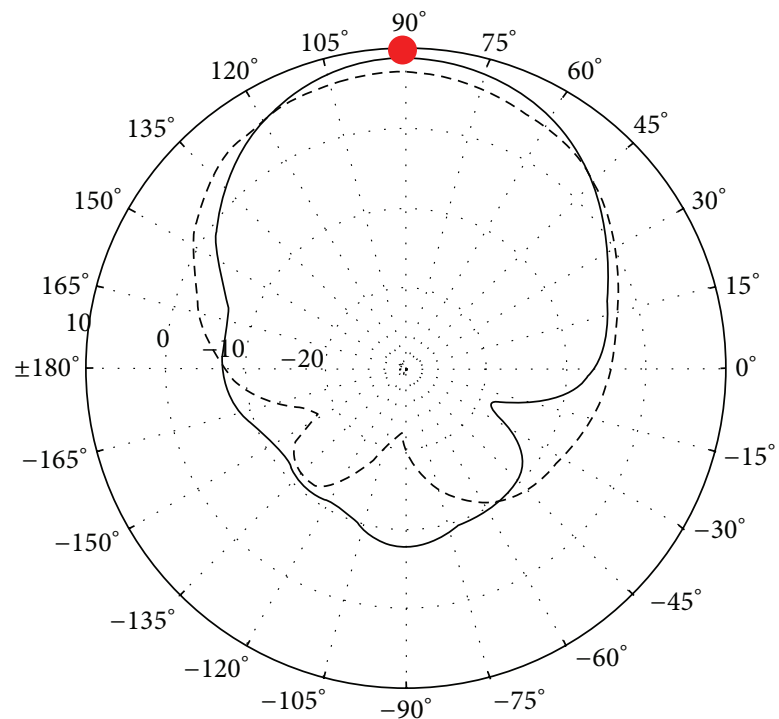

- Simulated

- - - Measured

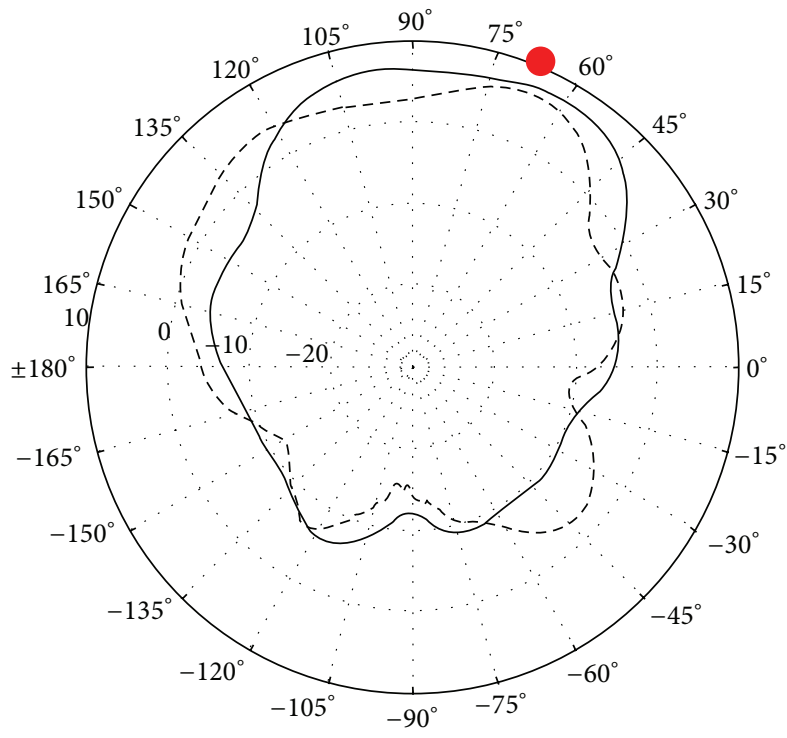

- Simulated

$$
\text { - - - Measured }
$$

(b)

(a)

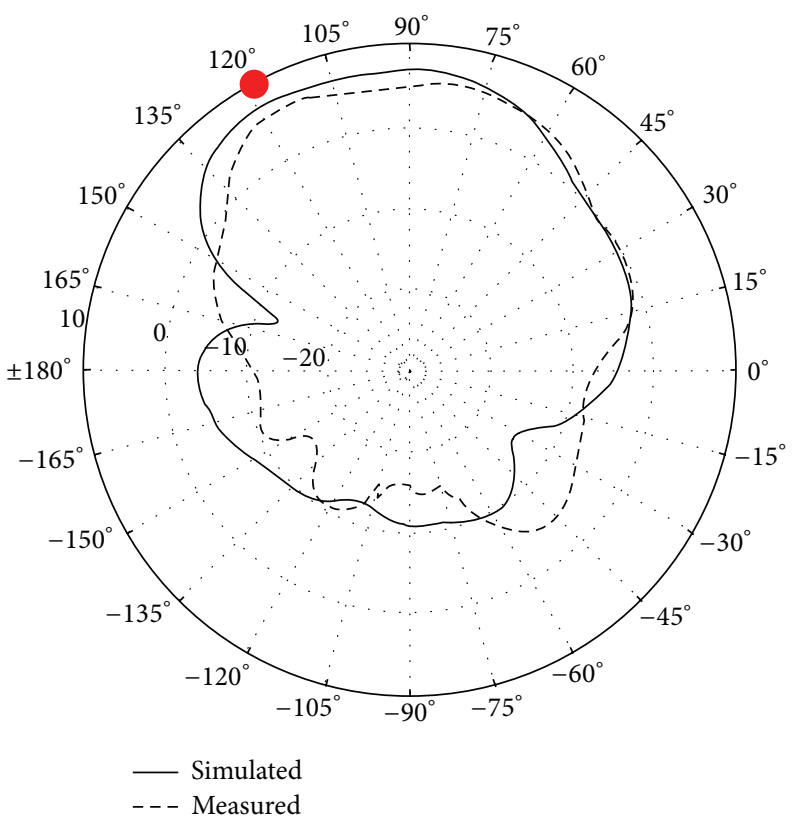

(c)

Figure 10: Comparison of measured and simulated 2D radiation patterns: (a) $\theta=90^{\circ}$ for $H_{01}=H_{02}=0$, (b) $\theta=64^{\circ}$ for $H_{01}=0$ and $H_{02}=0.19 \mathrm{~T}$, and (c) $\theta=118^{\circ}$ for $H_{01}=0.19 \mathrm{~T}$ and $H_{02}=0 \mathrm{~T}$.

\section{Acknowledgment}

The authors would like to acknowledge the support provided by the Deanship of Scientific Research at King Fahd University of Petroleum and Minerals (KFUPM) under Research Grant SB-121005.

\section{References}

[1] A. P. Feresidis, G. Goussetis, S. Wang, and J. C. Vardaxoglou, "Artificial magnetic conductor surfaces and their application to low-profile high-gain planar antennas," IEEE Transactions on Antennas and Propagation, vol. 53, no. 1, pp. 209-215, 2005. 
[2] J. Che and T. Kim, "Microstrip array antenna for PCs and IMT2000 base station," in Proceedings of the Conference: Microwave Conference, 2000 Asia-Pacific, pp. 25-28, 2000.

[3] C. A. Balanis, Antenna Theory: Analysis and Design, John Wiley \& Sons, Hoboken, NJ, USA, 3rd edition, 2005.

[4] C. Thongsopa, M. Krairiksh, N. Srirattana, and A. Intarapanich, "Single patch beam steering antenna," in Proceedings of the Asia-Pacific Microwave Conference, p. 37, Bangkok, Thailand, December 2000.

[5] S. Ha and C. W. Jung, "Single patch beam steering antenna with U-slot for wearable fabric applications," in Proceedings of the IEEE International Symposium on Antennas and Propagation and USNC/URSI National Radio Science Meeting, Washington, DC, USA, 2011.

[6] H. Attia, O. Siddiqui, and O. Ramahi, "Theoretical and experimental demonstration of beam steering of patch antenna with superstrate," in Proceedings of the IEEE Antennas and Propagation Society International Symposium (APSURSI '12), pp. 1-2, Chicago, Ill, USA, July 2012.

[7] W. Cao, Y. Xiang, B. Zhang, A. Liu, T. Yu, and D. Guo, "A low-cost compact patch antenna with beam steering based on CSRR-loaded ground," IEEE Antennas and Wireless Propagation Letters, vol. 10, pp. 1520-1523, 2011.

[8] S. S. I. Mitu, A. A. P. Gibson, M. Basorrah et al., "Analogue/digital ferrite phase shifter for phased array antennas," IEEE Antenna and Wireless Propagation Letters, vol. 9, 2010.

[9] S. Bae, Y.-K. Hong, J.-J. Lee et al., "Miniature and higher-order mode ferrite mimo ring patch antenna for mobile communication system," Progress In Electromagnetics Research B, no. 25, pp. 53-74, 2010.

[10] M. K. Meshram and B. R. Vishvakarma, "Scanning characteristics of ferrite fed microstrip array antenna," International Journal of Electronics, vol. 88, no. 5, pp. 549-559, 2001.

[11] S. Bae, Y. K. Hong, J. J. Lee et al., "Miniaturized broadband ferrite T-DMB antenna for mobile-phone applications," IEEE Transactions on Magnetics, vol. 46, no. 6, pp. 2361-2364, 2010.

[12] I. S. Nefedov, A.-C. Tarot, and K. Mahdjoubi, "Wire mediaferrite substrate for patch antenna miniaturization," in Proceedings of the IEEE International Workshop on Antenna Technology: Small and Smart Antennas Metamaterials and Applications (iWAT '07), pp. 101-104, March 2007.

[13] P. Parsons, K. Duncan, A. Giri, J. Q. Xiao, and S. P. Karna, "NiZn ferrite nanoparticles and their polymer composites for antenna miniaturization," in Proceedings of the 12th IEEE International Conference on Nano-Technology (NANO '12), pp. 1-5, IEEE, Birmingham, UK, August 2012.

[14] S. Bae, Y. K. Hong, and A. Lyle, "Effect of Ni-Zn ferrite on bandwidth and radiation efficiency of embedded antenna for mobile phone," Journal of Applied Physics, vol. 103, no. 7, Article ID 07E929, 2008.

[15] M. I. Kitra, C. J. Panagamuwa, P. McEvoy, J. C. Vardaxoglou, and J. R. James, "Low SAR ferrite handset antenna design," IEEE Transactions on Antennas and Propagation, vol. 55, no. 4, pp. 1155-1164, 2007.

[16] M. A. Amiri, C. A. Balanis, and C. R. Birtcher, "Gain and bandwidth enhancement of ferrite-loaded CBS antenna using material shaping and positioning," IEEE Antennas and Wireless Propagation Letters, vol. 12, pp. 611-614, 2013.

[17] L.-R. Tan, R.-X. Wu, C.-Y. Wang, and Y. Poo, "Ferrite-loaded SIW bowtie slot antenna with broadband frequency tunability," IEEE Antennas and Wireless Propagation Letters, vol. 13, pp. 325328, 2014.
[18] G. M. Yang, X. Xing, A. Daigle et al., "Electronically tunable miniaturized antennas on magnetoelectric substrates with enhanced performance," IEEE Transactions on Magnetics, vol. 44, no. 11, pp. 3091-3094, 2008.

[19] F. A. Ghaffar, J. R. Bray, and A. Shamim, "Theory and design of a tunable antenna on a partially magnetized ferrite LTCC substrate," IEEE Transactions on Antennas and Propagation, vol. 62, no. 3, pp. 1238-1245, 2014.

[20] N. Okamoto and D. Suzuki, "Electronic scanning by ferriteloaded corner reflector antennas," IEEE Transactions on Antennas and Propagation, vol. 32, no. 10, pp. 1013-1017, 1984.

[21] N. Apaydin, K. Sertel, and J. L. Volakis, "Non-reciprocal and magnetically scanned leaky-wave antenna using coupled CRLH lines," IEEE Transactions on Antennas and Propagation, vol. 62, no. 6, pp. 2954-2961, 2014.

[22] K. C. Hwang and H. J. Eom, "Ferrite-loaded groovy antenna," IEEE Transactions on Antennas and Propagation, vol. 53, no. 12, pp. 4172-4175, 2005. 

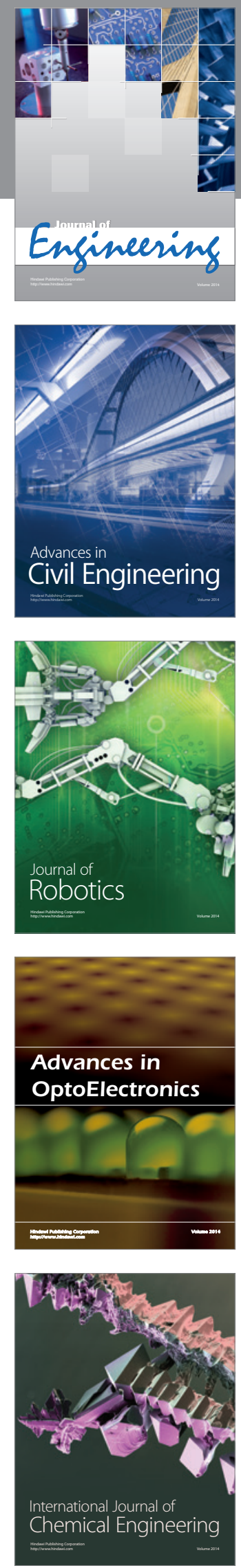

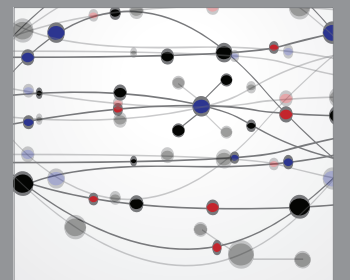

The Scientific World Journal
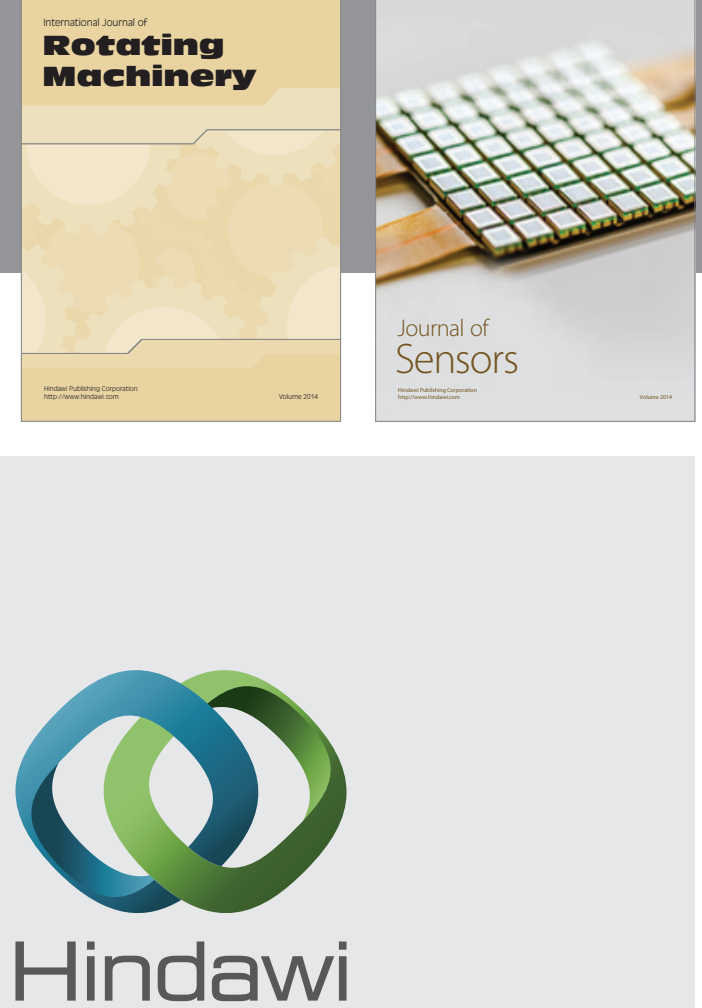

Submit your manuscripts at http://www.hindawi.com
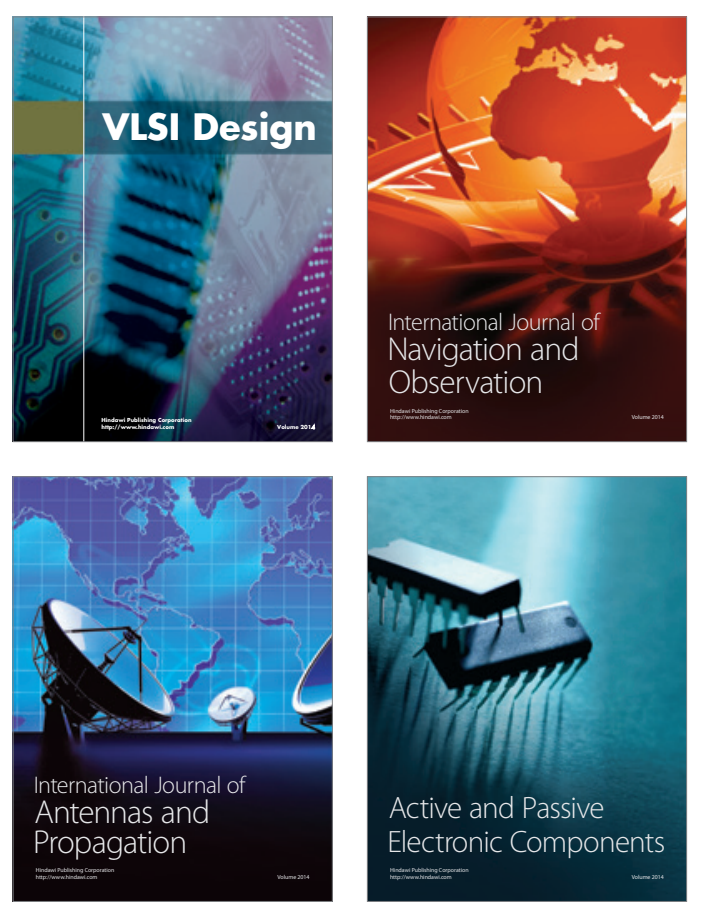
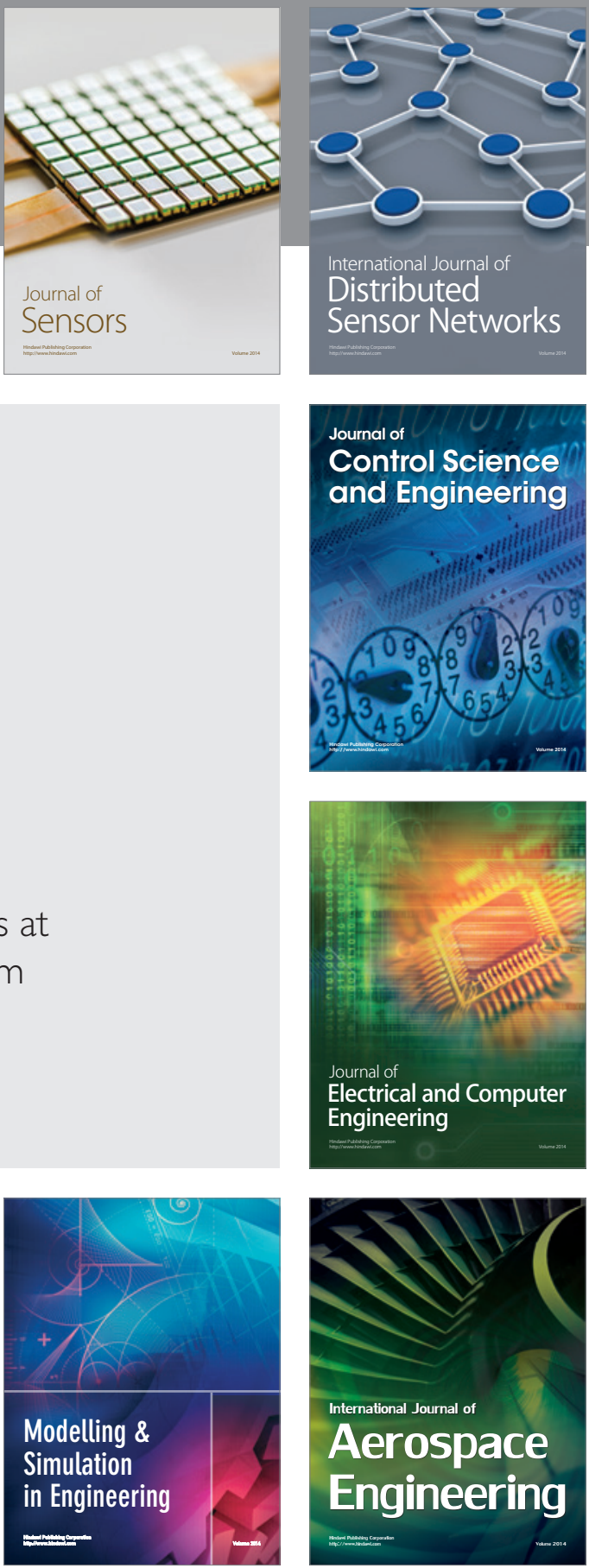

Journal of

Control Science

and Engineering
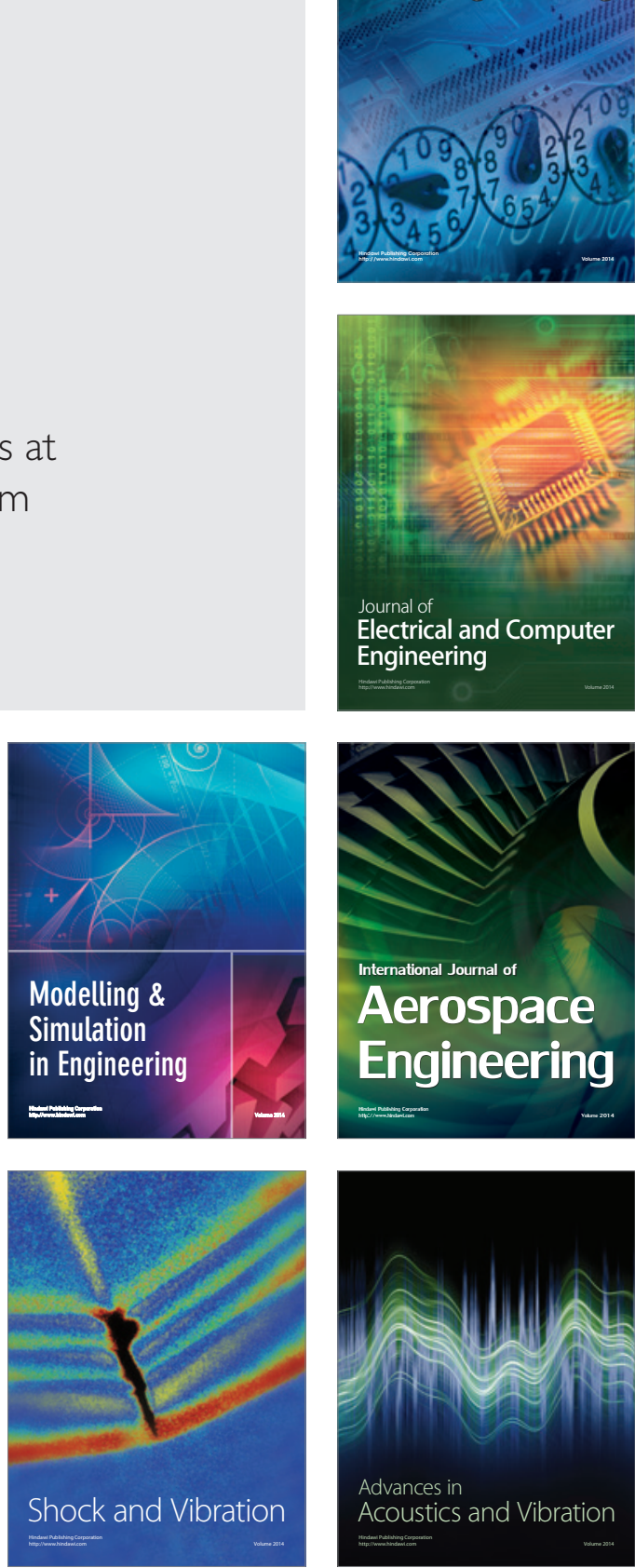